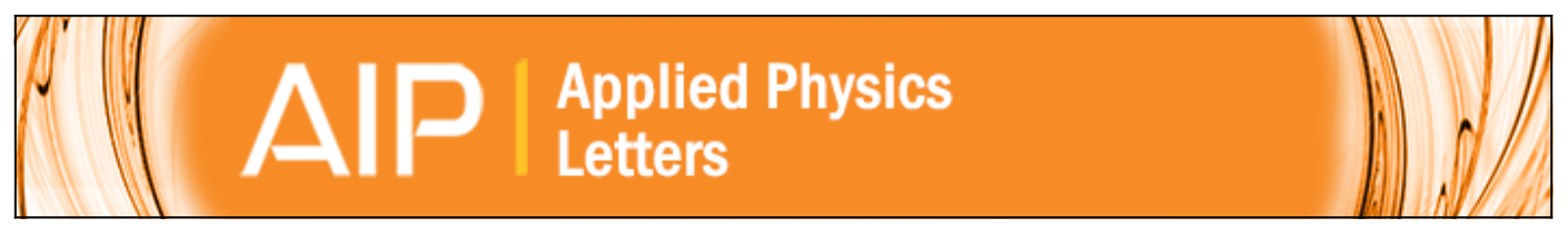

\title{
Ground-state modulation-enhancement by two-state lasing in quantum-dot laser devices
}

André Röhm, Benjamin Lingnau, and Kathy Lüdge

Citation: Applied Physics Letters 106, 191102 (2015); doi: 10.1063/1.4921173

View online: http://dx.doi.org/10.1063/1.4921173

View Table of Contents: http://scitation.aip.org/content/aip/journal/apl/106/19?ver=pdfcov

Published by the AIP Publishing

\section{Articles you may be interested in}

Stability of quantum-dot excited-state laser emission under simultaneous ground-state perturbation

Appl. Phys. Lett. 105, 191105 (2014); 10.1063/1.4901051

Comparison of dynamic properties of ground- and excited-state emission in p-doped InAs/GaAs quantum-dot lasers

Appl. Phys. Lett. 104, 181101 (2014); 10.1063/1.4875238

Effect of excited states on the ground-state modulation bandwidth in quantum dot lasers

Appl. Phys. Lett. 102, 191102 (2013); 10.1063/1.4804994

Ground-state power quenching in two-state lasing quantum dot lasers

J. Appl. Phys. 111, 043108 (2012); 10.1063/1.3682574

Simultaneous two-state lasing in quantum-dot lasers

Appl. Phys. Lett. 82, 1818 (2003); 10.1063/1.1563742
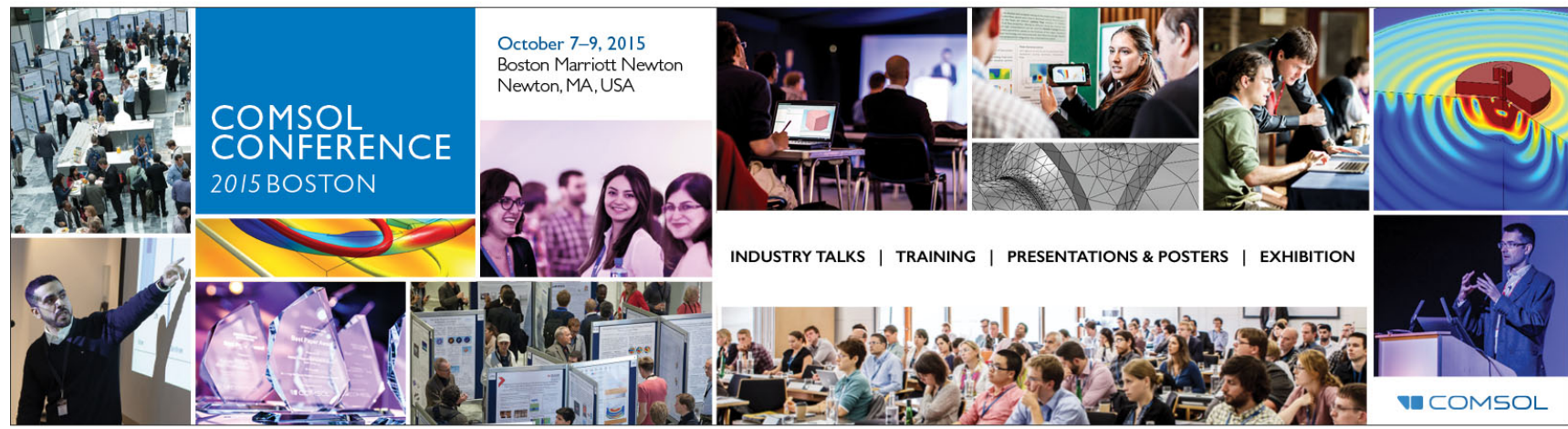


\title{
Ground-state modulation-enhancement by two-state lasing in quantum-dot laser devices
}

\author{
André Röhm, ${ }^{1}$ Benjamin Lingnau, ${ }^{1}$ and Kathy Lüdge ${ }^{2}$ \\ ${ }^{1}$ Institut für Theoretische Physik, Technische Universität Berlin, 10623 Berlin, Germany \\ ${ }^{2}$ Fachbereich Physik, Freie Universität Berlin, 14195 Berlin, Germany
}

(Received 11 March 2015; accepted 5 May 2015; published online 11 May 2015)

\begin{abstract}
We predict a significant increase of the $3 \mathrm{~dB}$-cutoff-frequency on the ground-state lasing wavelength for two-state-lasing quantum-dot lasers using a microscopically motivated multi-level rateequation model. After the onset of the second lasing line, the excited state acts as a high-pass filter, improving the ground-state response to faster modulation frequencies. We present both numerically simulated small-signal and large-signal modulation results and compare the performance of single and two-state lasing devices. Furthermore, we give dynamical arguments for the advantages of two-state lasing on data-transmission capabilities. (C) 2015 AIP Publishing LLC.
\end{abstract}

[http://dx.doi.org/10.1063/1.4921173]

The use of semiconductor laser devices for high-speed communication-network applications critically depends on the maximum achievable modulation frequency. A high bandwidth means faster data transmission and hence considerable effort has been put into improving the performance of lasing devices. ${ }^{1-3}$ Quantum-dot (QD) based devices were once predicted to have superior modulation capabilities when compared to quantum-well (QW) and bulk devices. ${ }^{4,5}$ However, the slow scattering dynamics are limiting the bandwidth of current QD based structures, so that they yet are unable to compete with high quality QW-based lasers. ${ }^{6-8}$

Nonetheless, due to their low threshold currents, high temperature stability, and high efficiency, ${ }^{9}$ studying their dynamic properties is still the topic of many recent publications. ${ }^{1,10-13}$ Moreover, QDs also exhibit some unique lasing behavior, setting them apart from other gain materials. Because of their effectively zero-dimensional structure, electronic states are quantized in a quasi-atom-like fashion, giving rise to multiple well separated energy levels. The lowest of these levels, the ground state (GS), is usually used for lasing purposes. However, because of the aforementioned slow scattering into the QD states, lasing on the first excited state (ES) is also obtainable. ${ }^{14-17}$ Pure ES lasers have been analyzed and have shown good modulation characteristics. ${ }^{16,18}$ The simultaneous lasing on the GS and ES is referred to as two-state lasing. ${ }^{19-22}$ In this paper, we study the interplay of two-state lasing and modulation capabilities. We go beyond studying only the effect of the excited states on the carrier lifetimes ${ }^{10-12}$ by also considering the effect of ES lasing, i.e., on the large and small-signal modulation response of the GS. To be able to correctly predict the performance, we use a microscopically motivated multi-level rate-equation model with stochastic spontaneous emission noise, which separately treats electron and hole dynamics. A first study of the effect of ES lasing on the relaxation oscillations with a simplified modeling approach was done in Ref. 23, however, without distinguishing between GS and ES photons as we do here. This letter is structured as follows: we first present the model used for numerical simulation and then present small and large-signal analysis of the two-state lasing simulations, before we summarize the results and conclude with the important implications of our findings.

The QD laser model is based on our previous models. ${ }^{17,24-26}$ We include the area density of GS and ES photons $N_{p h}^{m}$, with $m \in\{G S, E S\}$, which interact with the electron and hole occupation probabilities for GS and ES $\rho_{b}^{m}$, with $b \in\{e, h\}$. To account for the inhomogeneous broadening of the QD ensemble, we assume that only a fraction $f^{a c t}=0.366$ is optically active and a second set of variables $\rho_{b, i a}^{m}$ is introduced for the inactive fraction $\left(1-f^{a c t}\right)$. The effect of the inhomogeneous broadening on the scattering dynamics is therefore preserved. This approach is based on our previous works. ${ }^{17,25}$ Furthermore, the 2D carrier densities $w_{b}$ model the carrier reservoir corresponding to the QW layers surrounding the QDs. A sketch of the energetic structure can be seen in Fig. 1. The full set of 12 equations is given by

$$
\begin{aligned}
& \frac{d}{d t} N_{p h}^{m}=+\left(g_{m}\left[\rho_{e}^{m}+\rho_{h}^{m}-1\right]-\kappa_{m}\right) N_{p h}^{m} \\
&+\beta N^{Q D} f^{a c t} W_{m} \rho_{e}^{m} \rho_{h}^{m}, \\
& \frac{d}{d t} \rho_{b}^{m}=- \frac{g_{m}}{\nu_{m} N^{Q D} f a c t}\left(\rho_{e}^{m}+\rho_{h}^{m}-1\right) N_{p h}^{m} \\
&- W_{m} \rho_{e}^{m} \rho_{h}^{m}+R_{b, a}^{m}, \\
& \frac{d}{d t} \rho_{b, i a}^{m}=-W_{m} \rho_{e, i a}^{m} \rho_{h, i a}^{m}+R_{b, i a}^{m},
\end{aligned}
$$

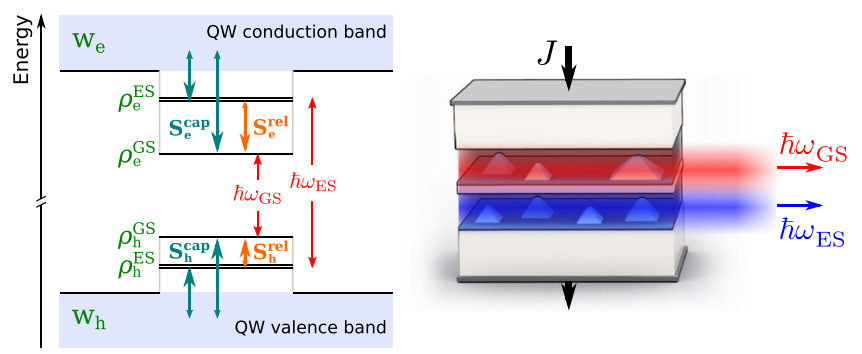

FIG. 1. Sketch of the energy levels and scattering channels of the QD model (left) and device structure (right). Both GS photons with $\omega_{G S}$ and ES photons with $\omega_{E S}$ are modeled. 


$$
\frac{d}{d t} w_{b}=+J-R_{\text {loss }}^{W} w_{e} w_{h}+R_{b}^{\text {well }} .
$$

Here, $g_{G S}=0.115 \mathrm{ps}^{-1}, g_{E S}=0.23 \mathrm{ps}^{-1}$, and $\kappa_{m}$, with $m$ $\in\{G S, E S\}$, denote the optical gain and losses, $N^{Q D}$ $=5 \times 10^{10} \mathrm{~cm}^{-2}$ is the area density of QDs per layer, $W_{G S}=0.44 \times 10^{-3} \mathrm{ps}^{-1}$ and $W_{E S}=0.55 \times 10^{-3} \mathrm{ps}^{-1}$ are the spontaneous emission rate coefficients, and $\nu_{m}$ is the degeneracy of the QD levels, which is 1 for the GS and 2 for the ES. $R_{\text {loss }}^{W}=0.09 \mathrm{~nm}^{2} \mathrm{~ns}^{-1}$ denotes the loss rate of QW carriers. The pump current density $J$ only enters in the QW equation, so that carriers have to scatter into the QD levels before interacting radiatively. This is described by the scattering terms $R_{b}^{m}$ for the QD equations

$$
\begin{aligned}
R_{b, a}^{m}= & S_{b, \text { in }}^{m, \text { cap }}\left(1-\rho_{b}^{m}\right)-S_{b, \text { out }}^{m, \text { cap }}\left(\rho_{b}^{m}\right) \\
& \pm \frac{1}{\nu_{m}}\left[S_{b, \text { in }}^{r e l}\left(1-\rho_{b}^{G S}\right) \rho_{b}^{E S}-S_{b, \text { out }}^{r e l} \rho_{b}^{G S}\left(1-\rho_{b}^{E S}\right)\right],
\end{aligned}
$$

where the $S$ coefficients are microscopically calculated scattering rates, ${ }^{24}$ which depend on QW densities and temperature $\left(S=S\left(w_{e}, w_{h}, T\right)\right)$. The scattering rates are calculated for an energetic depth of $64 \mathrm{meV}(40 \mathrm{meV})$ for the electron (hole) GS and $14 \mathrm{meV}(20 \mathrm{meV})$ for the ES. The coefficients are derived for Auger scattering, for further details, cf. Refs. 17, 24, and 27. The first line in Eq. (5) describes capture processes from the QW into the QD states, while the second line contains the intra-dot relaxation terms, where the plus (minus) sign applies to the GS (ES) equation. The inactive QDs experience identical scattering terms, but with all $\rho_{b}^{m}$ substituted by the corresponding $\rho_{b, i a}^{m}$. The QW scattering term $R^{\text {well }}$ is given by

$$
\begin{aligned}
R_{b}^{\text {well }}= & -2 N^{Q D} f^{a c t}\left[S_{b, \text { in }}^{G S, \text { cap }}\left(1-\rho_{b}^{G S}\right)-S_{b, \text { out }}^{G S, \text { cap }}\left(\rho_{b}^{G S}\right)\right] \\
& -4 N^{Q D} f^{a c t}\left[S_{b, \text { in }}^{E S, \text { cap }}\left(1-\rho_{b}^{E S}\right)-S_{b, \text { out }}^{E S, \text { cap }}\left(\rho_{b}^{E S}\right)\right] \\
& -2 N^{Q D}\left(1-f^{a c t}\right)\left[S_{b, \text { in }}^{G S, \text { cap }}\left(1-\rho_{b, \text { ia }}^{G S}\right)-S_{b, \text { out }}^{G S, \text { cap }}\left(\rho_{b, \text { ia }}^{G S}\right)\right] \\
& -4 N^{Q D}\left(1-f^{a c t}\right)\left[S_{b, \text { in }}^{E S, \text { cap }}\left(1-\rho_{b, \text { ia }}^{E S}\right)-S_{b, \text { out }}^{E S, \text { cop }}\left(\rho_{b, \text { ia }}^{E S}\right)\right] .
\end{aligned}
$$

Here, the prefactors in each line account for the degeneracy of the GS and ES, including spin degeneracy.

Isolating the effect of two-state lasing, i.e., the effect of additional ES photons in the cavity, requires to simulate two devices (with and without ES lasing) with approximately the same parameters. Thus, we need to keep as many parameters constant as possible. As introduced in Eq. (4), we employ two different loss terms, namely, $\kappa_{G S}$ and $\kappa_{E S}$, which we use to switch between two-state lasing and single-state lasing devices. Usually, the GS and ES will experience roughly the same optical losses $\kappa$ as their frequencies are relatively similar. However, the optical losses can become decoupled, e.g., when a dichroic mirror is considered. Dichroic mirrors have already been employed in experiments ${ }^{28,29}$ and use a coating that is reflective in the optical frequency of one state but transmissive in the other state.

For the parameters and scattering rates as used in this paper, the device achieves typical two-state lasing operation for $\kappa_{G S}=0.049 \mathrm{ps}^{-1}$ and $\kappa_{E S}=0.045 \mathrm{ps}^{-1}$. We simulate a second device structure by doubling the ES optical losses
$\kappa_{E S}$ to $0.09 \mathrm{ps}^{-1}$, therefore suppressing ES lasing. All other parameters and the scattering rates are identical. The lightcurrent characteristics for both cases are shown in the top panels of Fig. 2. We denote the GS threshold current density by $J_{t h}^{G S}$ and the ES threshold by $J_{t h}^{E S}$ (vertical lines in Fig. 2).

To obtain small-signal modulation bandwidths, the pump-current density was modulated in time in a harmonic fashion, i.e., $J(t)=J^{0}+\Delta J \sin (2 \pi f t)$. Here $J^{0}$ denotes the dc pump-current density, $f$ is the modulation frequency, and $\Delta J$ is the ac modulation amplitude of the current density, which was kept constant at $\Delta J \simeq 0.02 \times J_{t h}^{E S}$. The resulting modulation of photon densities is then normalized with respect to the zero-frequency modulation. Modulation response curves typically exhibit one resonance peak and then rapidly fall off towards high frequency (see Fig. 3). Thus, the maximum achievable modulation frequency is defined as the $3 \mathrm{~dB}$-cutoff-frequency $f_{-3 \mathrm{~dB}}$, i.e., where the normalized modulation amplitude falls below $-3 \mathrm{~dB} \simeq 0.5$.

The cutoff frequencies are shown for GS (red) and ES (blue) modulation in Figs. 2(c) and 2(d) for the two-state and single-state lasing case, respectively, as a function of the pump current density $J$. Additionally, this maximum bandwidth was also calculated for the total photon density (black), which corresponds to a measurement where ES and GS photons cannot be discerned (as, for instance, is done in Ref. 30). Light-colored parts correspond to modulation below lasing thresholds. There is a clear correlation between the lasing thresholds and cutoff-frequencies. Cutoff-frequencies are small below the GS threshold pump current density $J_{t h}^{G S}$ for both GS and ES and their shape is in accordance with simulations of Ref. 31. Above the GS threshold, cutofffrequencies are notably higher. While the GS cutofffrequency increases in a square-root-like fashion, the ES exhibits an immediate increase. However, the ES is not lasing at this point and therefore the output power is low. Also note that the GS modulation is independent of the optical

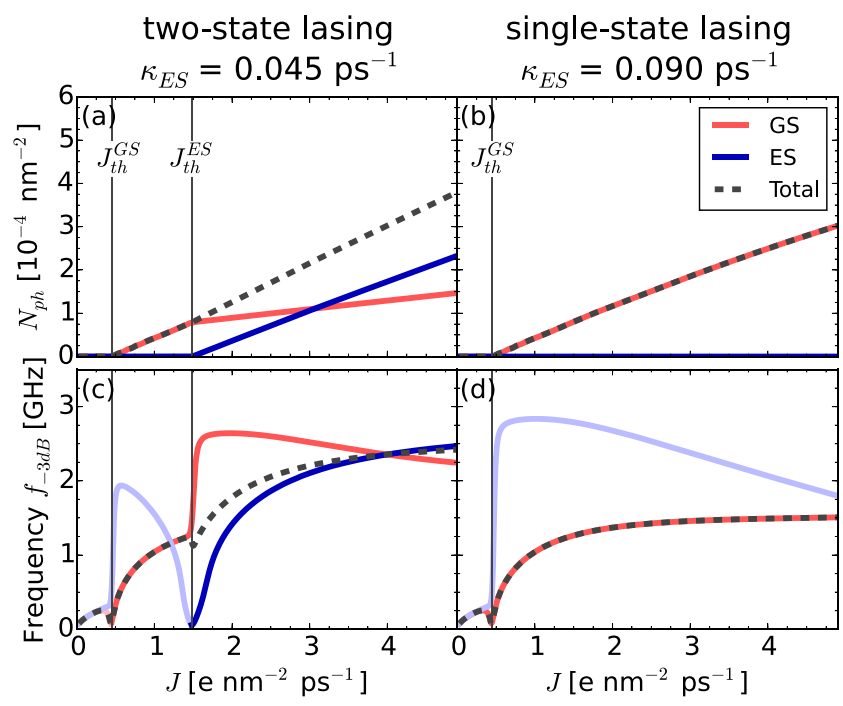

FIG. 2. (a) and (b) Simulated photon densities $N^{p h}$ and (c) and (d) small signal $3 \mathrm{~dB}$-cutoff-frequencies $f_{-3 \mathrm{~dB}}$ versus pump current density $J$ for GS (red) and ES (blue) emission. Left: Low ES losses $\kappa_{E S}=0.045 \mathrm{ps}^{-1}$; Right: high ES losses $\kappa_{E S}=0.09 \mathrm{ps}^{-1}$. Values for the combined light output of ES and GS are shown in black dashed. ES cutoff-frequency below threshold is shown in light blue. 
losses in the ES, as can be seen by comparing panels (c) and (d) for currents below the ES threshold.

Dynamically, the most interesting feature is the reaction of the GS modulation bandwidth to the appearance of the ES lasing: Above the ES threshold current density $J_{t h}^{E S}$, the GS cutoff-frequency more than doubles. This is true for all parameter and scattering rate sets that were tested and is consistently reappearing whenever two-state lasing starts, as long as different nonlinear rates for electrons and holes are used. Also, close to the ES threshold, the ES modulation frequency is reaching a minimum (Fig. 2(c)). This is related to the undamped relaxation oscillations, which suppress any high-frequency modulation by their long transient times. Furthermore, relaxation oscillation frequencies are minimal at threshold, so that resonances cannot be exploited. Above threshold, the ES cutoff-frequencies increase in a similar fashion to the GS above its threshold.

The modulation bandwidth of the total photon intensity (black lines in Figs. 2(c) and 2(d)) also shows a slight increase in the modulation bandwidth for two-state lasing currents. This has already been noted in the literature and has been seen in experiments. ${ }^{30}$ In this regard, the results of Abusaa et al. $^{23}$ should also be noted. They calculated the relaxation oscillation frequency for a two-state lasing device, but only considering the total intensity and not distinguishing between ES and GS photons. Their results correctly predict the numerically obtained modulation frequencies for the total photon density presented here.

To understand the GS modulation bandwidth enhancement, i.e., the sudden increase in the GS cutoff-frequency upon the onset of two-state lasing in the small-signal analysis, we present two sets of modulation response curves in Fig. 3. The GS (red) and ES (blue) modulation response is shown versus modulation frequency in a double-logarithmic plot for current densities slightly below (a) and slightly above (b) the ES threshold $J_{t h}^{E S}$. Note that we chose not to normalize the modulation response in Fig. 3. Below the ES threshold (a), neither the GS nor the ES shows a high cutofffrequency, as indicated by the thin black lines, and no resonance peaks can be seen. Further, the ES modulation is much smaller than that of the GS, as the ES is not lasing yet and accordingly has a small response to external periodic signals.

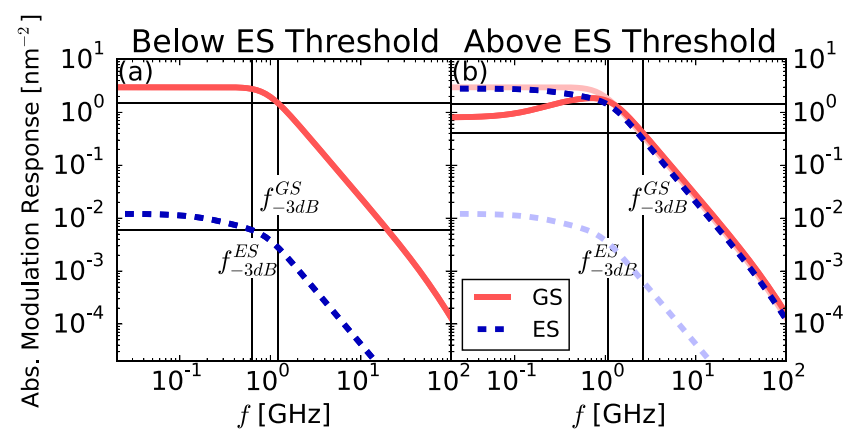

FIG. 3. Photon density $N_{p h}^{m}$ modulation response (not normalized) for GS (red) and ES (blue) versus driving frequency $f$ are shown for pump current densities below ES threshold $J \simeq 0.9 \times J_{\text {th }}^{E S}$ (a) and above ES threshold $J \simeq$ $1.2 \times J_{t h}^{E S}$ (b) for the two-state lasing device. Light-colored lines in (b) are reproductions of the lines in (a) for better comparison.
Fig. 3(b) shows modulation response curves above the ES threshold. The light-colored lines are reproductions of panel (a) for better comparison. It can be seen that the highfrequency flank of the GS response does not change when two-state lasing starts. In contrast, the GS modulation response (red) towards low frequencies is reduced compared to sub-ES threshold currents (light red). As the $3 \mathrm{~dB}$-cutofffrequency is defined with respect to the low-frequency modulation amplitude, i.e., for a normalized response curve, this drop in low-frequency modulation response increases the cutoff-frequency of the GS.

The drastic increase in the GS small signal bandwidth at $J_{\text {th }}^{E S}$ of Fig. 2(c) can therefore be linked to the decreasing low-frequency response of the absolute GS modulation response, which changes the normalization level, while the high-frequency GS response is unaffected. The lower the small-frequency modulation falls, the further out the cutoff-frequency will be pushed by this renormalization and hence the larger the small-signal bandwidth $f_{-3 \mathrm{~dB}}$ becomes. The limit of this small-frequency GS modulation response for $f \rightarrow 0 \mathrm{~Hz}$ can always be directly inferred from the lightcurrent characteristic of the device. When the system is driven by a slowly varied current, i.e., oscillations with small driving frequency $f$, the laser will reach its steady state adiabatically. Then, the absolute modulation response will be given by the differential change in the photon density $\Delta N_{p h}=N_{p h}(J+\Delta J)-N_{p h}(J-\Delta J)$. For a small modulation current $\Delta J$, this can be expressed as $\Delta N_{p h} \simeq\left(\frac{d}{d J} N_{p h}\right) 2 \Delta J$, where the slope of the light-current curve $\frac{d}{d J} N_{p h}$ was used. As two-state lasing results in a smaller GS slope after ES threshold, the cutoff-frequency $f_{-3 \mathrm{~dB}}$ increases.

Therefore, if we can change the slope of the GS lightcurrent curve above ES threshold $J_{t h}^{E S}$, e.g., by manipulating the scattering rates, we can manufacture very high cutofffrequencies in the GS response. However, simultaneously, GS large signal modulation becomes increasingly difficult if the GS slope tends to zero, as different current densities will result in almost identical light output. Therefore, the smallsignal modulation bandwidth alone is not a good prediction for large signal modulation capabilities once two-state lasing occurs, as a trade-off between maximum frequency and absolute photon density modulation has to be taken into account. Note that a separate description of electrons and holes and the carrier-density dependent scattering rates are required to describe the two-state lasing correctly, ${ }^{17}$ while simpler models would result in a constant GS power after the ES threshold. The modulation response of the ES (blue lines in Fig. 3), on the other hand, does not change qualitatively when comparing above and below $J_{t h}^{E S}$. In terms of absolute modulation response, it is shifted towards higher photon densities, but $f_{3 d B}^{E S}$ stays minimal.

Modulation bandwidths are also strongly dependent on the scattering scheme, as was reported in Ref. 12. The authors found indirect, ES-mediated capture processes to be detrimental to GS bandwidth. As is detailed in Refs. 17 and 14, two-state lasing can only be achieved by the decoupling of GS and ES carrier levels because of the delayed scattering scheme. To this end, our findings suggest that the indirect capture can also be used to increase bandwidth, namely, by achieving two-state lasing. 
To perform a realistic large-signal modulation, we take into account stochastic spontaneous emission by the inclusion of a Gaussian white noise term in the photon equation of (4), the derivation of which can be found in Ref. 32. We simulated large-signal modulation of non-return-to-zero onoff-keyed pseudo-random bit sequences for the single-state and two-state lasing device. We modulated the current density between $J_{\text {low }}=1.7 \times J_{\text {th }}^{E S}$ and $J_{\text {high }}=2 \times J_{t h}^{E S}$. A bit series with a bit rate of $f$ and rise/fall times for the current signal of 50 ps between different bits is considered.

The resulting eye-diagrams for $f=4 \mathrm{GBit} / \mathrm{s}$ are shown in Fig. 4. We show this as an example, but the qualitative results remain unchanged for higher modulation frequency and contrast. The GS response in the two-state lasing case in Fig. 4(a) shows clear open eyes, while the ES suffers from patterning effects (Fig. 4(c)). Upon switching to the singlestate lasing device, the GS performance deteriorates (Fig. 4(b)), leading to weaker eye-opening. Naturally, the ES is turned off for the single-state lasing case and therefore no modulation was obtained in Fig. 4(d). The cleaner modulation obtained for the GS during two-state lasing can be linked to the high cutoff-frequency seen in the small-signal analysis of Fig. 2(c), which in turn is related to the damped modulation response for low frequencies as seen in Fig. 3(b). On the other hand, the spontaneous emission noise is slightly more pronounced due to the lower absolute intensity modulation. Overall, this behavior implies that the appearance of ES lasing improves the high-frequency modulation of the GS.

From our numerical simulations for small and largesignal modulation, we conclude that two-state lasing effectively damps low-frequency modulations, i.e., long transients that would destroy clean modulation, while still permitting high-frequency modulation. Therefore, the effect of ES lasing on GS lasing is best described as a high-pass filter. The ES photons act as a buffer for ES carrier levels, due to gain clamping: Higher pump currents will only increase the ES photon densities, not the ES carrier occupations. Therefore, when excitation is slow, i.e., $f$ small, the ES occupations, and thus the net charge-carrier relaxation from the ES to the GS, stay approximately constant. The transmission of low

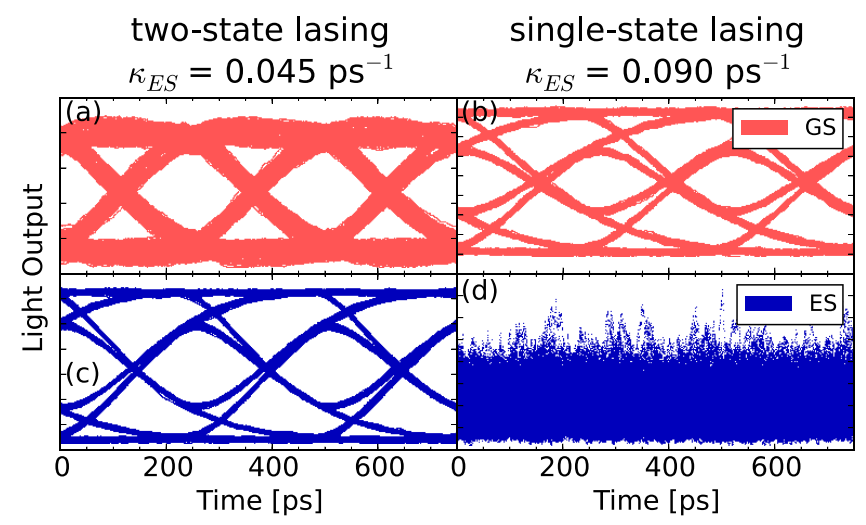

FIG. 4. Eye diagrams for GS ((a) and (b) red) and ES ((c) and (d) blue) modulation for low ES losses $\kappa_{E S}=0.045 \mathrm{ps}^{-1}$ ((a) and (c)) and high ES losses $\kappa_{E S}=0.09 \mathrm{ps}^{-1}$ ((b) and (d)). Note, in (d), the ES does not reach lasing and therefore no ES modulation is achieved. The current density was randomly varied between $J_{\text {low }} \simeq 1.7 \times J_{\text {th }}^{E S}$ and $J_{\text {high }} \simeq 2 \times J_{\text {th }}^{E S}$ at a frequency of 4 GBit/s. frequency modulation to the GS is thus reduced. In contrast, high frequencies are still able to reach the GS, when the ES carrier and photon levels cannot equilibrate fast enough to insulate GS carrier levels.

Nevertheless, the GS exhibits nonzero modulation at low frequencies due to the direct capture of charge carriers from the QW to the GS, which is, however, slower than the relaxation. Furthermore, the relaxation rates also depend on the QW densities, leading to a modulation of the GS even for clamped ES occupation. As the relaxation is, however, the dominating contribution to the GS carrier in-scattering, we still see an improved GS performance during two-state lasing in our large-signal modulation eye-diagrams. Therefore, this effect is promising for application purposes and merits thorough experimental investigation. We note that an increase in GS performance, similar to our prediction, was already experimentally observed in Ref. 30. However, the authors switched between two-state and single-state lasing by increasing the temperature, which might have decreased the modulation improvement.

We simulated two-state lasing QD devices and their modulation properties. We report an abrupt increase in the GS small-signal modulation bandwidth at the onset of twostate lasing, which is accompanied by the sudden appearance of a resonance peak in the modulation curve. This is also visible in the large-signal modulation eye diagrams, where the GS shows an improved performance. When investigating this phenomenon, we found that the interplay of ES photons and ES carriers effectively limits the influence of slow modulation frequencies on the GS by ES clamping. However, this breaks down when modulation frequency becomes too fast for the ES to reach its equilibrium. Hence, high frequencies suitable for fast switching in data transmission applications are still able to reach the GS, while slow transients are effectively filtered out by the ES, improving the GS response. In this context, the slope of the GS light-current curve and thus the carrier transfer into the GS during twostate lasing is important. This can only be predicted correctly by using a model with separate electron and hole densities, direct GS capture and QW dependent scattering rates.

The parameters used in our simulations were not optimized to yield maximum bandwidth but rather to clearly present the effect of two-state lasing. From our theoretical analysis, we can expect even devices that are already fast to profit from two-state lasing. If the slope of the GS in the light-current characteristic becomes smaller, the GS cutofffrequencies can reach higher values. However, the largesignal modulation will become impossible in the limit of a saturated GS intensity. Furthermore, a larger ES intensity slope leads to more energy being lost as ES photons, and followingly a lower efficiency. Therefore, a tradeoff between these different trends has to be taken into account.

We would like to thank L. Jaurigue for fruitful discussions and advice. This work was supported by SFB 787 .

${ }^{1}$ D. Gready, G. Eisenstein, V. Ivanov, C. Gilfert, F. Schnabel, A. Rippien, J. P. Reithmaier, and C. Bornholdt, IEEE Photonics Technol. Lett. 26(1), 11-13 (2014).

${ }^{2}$ S. Bhowmick, M. Baten, T. Frost, B. S. Ooi, and P. Bhattacharya, IEEE J. Quantum Electron. 50(1), 7 (2014). 
${ }^{3}$ O. Mollet, A. Martinez, K. Merghem, S. Joshi, J. G. Provost, F. Lelarge, and A. Ramdane, Appl. Phys. Lett. 105, 141113 (2014).

${ }^{4}$ Y. Arakawa and H. Sakaki, Appl. Phys. Lett. 40, 939 (1982).

${ }^{5}$ M. Asada, Y. Miyamoto, and Y. Suematsu, IEEE J. Quantum Electron. 22(9), 1915-1921 (1986).

${ }^{6}$ M. Sugawara, N. Hatori, M. Ishida, H. Ebe, Y. Arakawa, T. Akiyama, K. Otsubo, T. Yamamoto, and Y. Nakata, J. Phys. D 38, 2126-2134 (2005).

${ }^{7}$ C. Wang, F. Grillot, and J. Even, IEEE J. Quantum Electron. 48(9), 1144-1150 (2012).

${ }^{8}$ F. Lelarge, B. Dagens, J. Renaudier, R. Brenot, A. Accard, F. van Dijk, D. Make, O. L. Gouezigou, J. G. Provost, F. Poingt, J. Landreau, O. Drisse, E. Derouin, B. Rousseau, F. Pommereau, and G. H. Duan, IEEE J. Sel. Top. Quantum Electron. 13(1), 111 (2007).

${ }^{9}$ D. Bimberg, Electron. Lett. 44, 168 (2008).

${ }^{10}$ B. Lingnau, K. Lüdge, W. W. Chow, and E. Schöll, Appl. Phys. Lett. 101(13), 131107 (2012).

${ }^{11}$ M. Gioannini and M. Rossetti, IEEE J. Sel. Top. Quantum Electron. 17(5), 1318-1326 (2011).

${ }^{12}$ Y. Wu, R. A. Suris, and L. V. Asryan, Appl. Phys. Lett. 102, 191102 (2013).

${ }^{13}$ D. Gready and G. Eisenstein, IEEE J. Sel. Top. Quantum Electron. 19(4), 1900307 (2013).

${ }^{14}$ A. Markus, J. X. Chen, C. Paranthoen, A. Fiore, C. Platz, and O. GauthierLafaye, Appl. Phys. Lett. 82(12), 1818 (2003).

${ }^{15}$ F. Grillot, N. A. Naderi, J. B. Wright, R. Raghunathan, M. T. Crowley, and L. F. Lester, Appl. Phys. Lett. 99, 231110 (2011).

${ }^{16} \mathrm{C}$. Wang, B. Lingnau, K. Lüdge, J. Even, and F. Grillot, IEEE J. Quantum Electron. 50(9), 723-731 (2014).

${ }^{17}$ A. Röhm, B. Lingnau, and K. Lüdge, IEEE J. Quantum Electron. 51(1), 2000211 (2015).

${ }^{18}$ D. Arsenijević, A. Schliwa, H. Schmeckebier, M. Stubenrauch, M. Spiegelberg, D. Bimberg, V. Mikhelashvili, and G. Eisenstein, Appl. Phys. Lett. 104, 181101 (2014).
${ }^{19}$ E. A. Viktorov, P. Mandel, J. Houlihan, G. Huyet, and Y. Tanguy, Appl. Phys. Lett. 87(5), 053113 (2005).

${ }^{20}$ M. Sugawara, N. Hatori, H. Ebe, M. Ishida, Y. Arakawa, T. Akiyama, K. Otsubo, and Y. Nakata, J. Appl. Phys. 97, 043523 (2005).

${ }^{21}$ V. V. Korenev, A. V. Savelyev, A. E. Zhukov, A. V. Omelchenko, and M. V. Maximov, Semiconductors 47(10), 1397-1404 (2013).

${ }^{22}$ M. Gioannini, D. Dommermuth, L. Drzewietzki, I. Krestnikov, D. Livshits, M. Krakowski, and S. Breuer, Opt. Express 22(19), 23402 (2014).

${ }^{23}$ M. Abusaa, J. Danckaert, E. A. Viktorov, and T. Erneux, Phys. Rev. A 87, 063827 (2013).

${ }^{24}$ N. Majer, S. Dommers-Völkel, J. Gomis-Bresco, U. Woggon, K. Lüdge, and E. Schöll, Appl. Phys. Lett. 99, 131102 (2011).

${ }^{25}$ B. Lingnau, W. W. Chow, E. Schöll, and K. Lüdge, New J. Phys. 15, 093031 (2013).

${ }^{26}$ B. Lingnau, W. W. Chow, and K. Lüdge, Opt. Express 22(5), 4867-4879 (2014).

${ }^{27}$ Nonlinear Laser Dynamics - From Quantum Dots to Cryptography, edited by K. Lüdge (WILEY-VCH, Weinheim, 2012), Chap. 1, pp. 3-34.

${ }^{28}$ Y. Kaptan, H. Schmeckebier, B. Herzog, D. Arsenijević, M. Kolarczik, V. Mikhelashvili, N. Owschimikow, G. Eisenstein, D. Bimberg, and U. Woggon, Appl. Phys. Lett. 104, 261108 (2014).

${ }^{29}$ Y. Kaptan, A. Röhm, B. Herzog, B. Lingnau, H. Schmeckebier, D. Arsenijević, V. Mikhelashvili, O. Schops, M. Kolarczik, G. Eisenstein, D. Bimberg, U. Woggon, N. Owschimikow, and K. Lüdge, Appl. Phys. Lett. 105, 191105 (2014).

${ }^{30}$ P. F. Xu, T. Yang, H. M. Ji, Y. L. Cao, Y. X. Gu, Y. Liu, W. Q. Ma, and Z. G. Wang, J. Appl. Phys. 107(1), 013102 (2010).

${ }^{31}$ M. Lorke, T. Suhr, N. Gregersen, and J. Mørk, Phys. Rev. B 87, 205310 (2013).

${ }^{32}$ S. Wilkinson, B. Lingnau, J. Korn, E. Schöll, and K. Lüdge, IEEE J. Sel. Top. Quantum Electron. 19(4), 1900106 (2013). 